\title{
Photocatalytic C-N addition amination of olefin
}

\author{
Shihao Zhu ${ }^{1}$, Keran Chen ${ }^{1}$ and Xinrui Zhou*1 \\ ${ }^{1}$ Department of Fine Chemicals, Dalian University of Technology, Dalian, China
}

\begin{abstract}
Amines widely exist in nature, wherein some of cyclic amines have crucially biological and physiological activities. They are also the basic blocks for the syntheses of important intermediates, raw materials or fine chemicals such as pharmaceuticals, pesticides and dyes. Amine synthesis by photocatalytic amination of phthalimide and olefins using porphyrin iron (III) or phthalocyanine iron(II) as photosensitizer was investigated in this paper. The results showed that the reactions selectively developed C-N addition and generated a series of compounds containing benzazepine structure with the highest yield of $99.0 \%$ at $30{ }^{\circ} \mathrm{C}$. The scope of substrates was further examined and the results demonstrated that the reaction was effective to various olefins, especially to aliphatic olefins. In addition, the influence of the light source was further investigated. The simple, low-power, insect trap lamp (6 W, $365 \mathrm{~nm})$ was used as the light source for the reaction, and a high yield of $95.0 \%$ was obtained. Therefore, the inspiring results provide a green and efficient method for the synthesis of compounds containing benzazepine structure.
\end{abstract}

\section{Introduction}

Amines widely exist in nature, wherein some of cyclic amines have crucially biological and physiological activities $[1,2]$. They are also the basic blocks for the syntheses of important intermediates, raw materials or fine chemicals such as pharmaceuticals, pesticides and dyes [4-6]. Many methods have been applied to synthesize amines [7-11], such as Hoffmann amide degradation, nitro compound reduction, aldehyde or ketone hydroamination, nitrile hydrogenation and addition amination. Among these synthetic methods, addition amination [9] is one of the most direct and effective methods. By addition amination, ammonia or amines can be added directly to double or triple bonds to form new organic amines, hence compared with other amination methods, addition amination displays various advantages like the most theoretical atom utilization, short reaction procedure; positional selectivity and stereoselectivity. The inner molecular cyclization can be completed by this method to produce nitrogencontaining heterocyclic compounds. Therefore, addition amination plays an important role in the field of total synthesis of natural products, pharmaceutical molecules and preparation of fine chemical materials.

It has been reported that $o$-phthalimide (PHT) was token use as both photosensitizer and nitrogencontaining source for preparation of primary amine under high-pressure Hg-lamp, however light efficiency was too low that cause reaction time seriously delayed and many by-side products generated [12]. In this paper, more efficient photosensitizer, porphyrin iron and phthalocyanine iron were introduced. Metal porphyrins were wide employed as the chlorophyll model to sucessfully develop the artificial photosynthesis.
Improved by the success, we tested PHT addtion amination in the presence of phthalocyanine iron (II) $(\mathrm{PcFe})$, tetrasulfophthalocyanine iron (II) $(\mathrm{SxPcFe})$, meso-tetrakisphenylporphyrin iron (III) chloride (TPPFeCl).

The results showed that the reactions selectively developed $\mathrm{C}-\mathrm{N}$ addition (Fig. 1). The mildest reaction using simple, low-power, insect trap lamp365 (6 W, 365 $\mathrm{nm}$ ) as the light source generated C-N addtion product in $95.0 \%$ yield at $30{ }^{\circ} \mathrm{C}$. Therefore, photocatalytic C-N addition amination of olefin develpoed here provides an inspriring way toward the amines containing benzazepine.

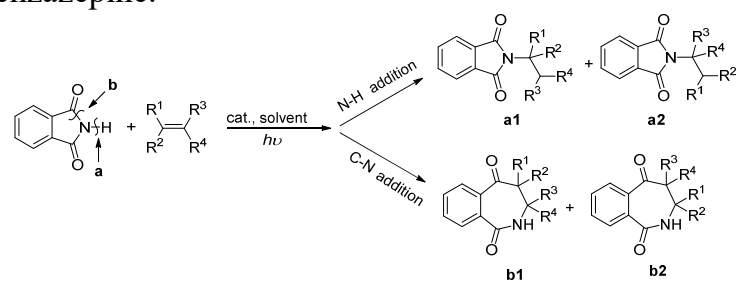

Fig. 1. Photocatalytic addition amination of olefin.

\section{Materials and Instrument}

$\mathrm{TPPFeCl}, \mathrm{PcFe}, \mathrm{SxPcFe}$ were synthesized according to the references reported $[13,14]$. Other reagents were obtained commercially.

$1 \mathrm{H}(400 \mathrm{MHz})$ and $13 \mathrm{C}(100 \mathrm{MHz})$ NMR-spectra were recorded on Bruker Ultra-Shield spectrometers in $\mathrm{CDCl}$. High-pressure mercury lamp (UV-lamp/250-450, Giguang, 250 watt, $250-450 \mathrm{~nm})$, residentially germicidal ultraviolet lamp (UV-Lamp-254, Philips, $6 \mathrm{~W}$, $254 \mathrm{~nm}$,) and insect trap lamp (UV-Lamp-365, Philips, 6 $\mathrm{W}, 365 \mathrm{~nm})$ were obtained commercially. High

\footnotetext{
* Corresponding author: xinrui@dlut.edu.cn
} 
Performance Liquid Chromatography (HPLC) was performed on an Agilent HP 1100 chromatograph equipped with a DAD detector using a fused XB-C18 capillary column (Welch Ultimate, $25.0 \mathrm{~cm} \times 4.6 \mathrm{~mm} \times$ $5.0 \mu \mathrm{m})$. A standard program was used for all HPLC analysis, i. e. eluent concentration of acetonitrile water solution was increased from the initial concentration $30 \%$ to $100 \%$ with $3.5 \%$ per minute, eluent flow rate was $0.8 \mathrm{ml} / \mathrm{min}$, and monitoring wavelength was at 220 $\mathrm{nm}$ and $254 \mathrm{~nm}$.

\section{Results \& Discussion}

At first, cyclohexene was used as the substrate in the experiment, which was carried out according to the reported reaction procedure but with the addition of photosensitive agent TPPFeCl. Unexpected results were found in the experiment. After various spectral analysis and comparison with the standard spectra, the product under certain conditions was identified as the cyclic expansion product from $\mathrm{C}-\mathrm{N}$ addition. The product yield and selectivity were $99.0 \%$ (Table 1, Entry 1) by HPLC spectroscopy. The experiment has been repeated many times and the reproducibility is indentical. Such high selectivity and yield have not been reported so far. Then, the optimal reaction conditions were obtained by optimizing the reaction conditions.

Conditional experiments were carried out first in the absence of $\mathrm{N} 2$, isolated yield was only $56.8 \%$ and the selectivity was $73.2 \%$ (Entry 2). In the absence of porphyrin photosensitizers, isolated yields were $67.2 \%$ and $65.2 \%$ respectively, and the selectivity was $79.6 \%$ and 74.0 (Entry 3,4). The experiments using PcFe and PcSxFe had little reaction (Entry 5,6). NaOH instead of for triethylamine (TEA) generated only a small amount of product (Entry 7). The same base effect can be achieved by using diisopropylamine (DIPA) besides of TEA (Entry 8), but almost no reaction can be achieved in experiments without base (Entry 9) and no reaction can be achieved in experiments without porphyrins and bases (Entry 10). Light, porphyrins, high sterically hindered amines and inert gases are the four necessary conditions for this type reaction.

Table 1. Condition experiment of photocatalytic addition amination*.

\begin{tabular}{|c|c|c|c|c|c|c|c|c|c|}
\hline Entry & Olefin & Catalyst & $h v / \lambda(\mathrm{nm}) / \mathrm{Watt}$ & Base & $\mathrm{N}_{2}$ & $\begin{array}{l}\text { Time } \\
\text { (h) }\end{array}$ & $\begin{array}{c}\text { Conversion } \\
(\%) \\
\end{array}$ & $\begin{array}{c}\text { Selectivity } \\
(\%)\end{array}$ & $\begin{array}{c}\text { Yeild } \\
(\%)\end{array}$ \\
\hline 1 & 1 & TPPFeCI & UV-lamp/250-450/250 & TEA & Yes & 2 & 99.0 & 99.9 & 99.0 \\
\hline 2 & 1 & TPPFeCI & UV-lamp/250-450/250 & TEA & No & 2 & 77.6 & 73.2 & 56.8 \\
\hline 3 & 1 & - & UV-lamp/250-450/250 & $\mathrm{NaOH}$ & Yes & 2 & 84.4 & 79.6 & 67.2 \\
\hline 4 & 1 & - & UV-lamp/250-450/250 & TEA & Yes & 2 & 88.0 & 74.0 & 65.2 \\
\hline 5 & 1 & PcFe & UV-lamp/250-450/250 & TEA & Yes & 2 & 0 & - & 0 \\
\hline 6 & 1 & PeSxFe & UV-lamp/250-450/250 & TEA & Yes & 2 & - & - & trace \\
\hline 7 & 1 & TPPFeCI & UV-lamp/250-450/250 & $\mathrm{NaOH}$ & Yes & 2 & - & - & trace \\
\hline 8 & 1 & TPPFeCI & UV-lamp/250-450/250 & DIPA & Yes & 2 & 99.0 & 99.9 & 99.0 \\
\hline 9 & 1 & TPPFeCI & UV-lamp/250-450/250 & - & Yes & 2 & 0 & - & 0 \\
\hline 10 & 1 & - & UV-lamp/250-450/250 & - & Yes & 2 & 0 & - & 0 \\
\hline 11 & 2 & TPPFeCl & UV-lamp/250-450/250 & TEA & Yes & 2 & 95.5 & 97.5 & 93.1 \\
\hline 12 & 3 & TPPFeCI & UV-lamp/250-450/250 & TEA & Yes & 2 & 96.6 & 97.6 & 94.3 \\
\hline 13 & 4 & TPPFeCI & UV-lamp/250-450/250 & TEA & Yes & 5 & 40.5 & 88.7 & 35.9 \\
\hline 14 & 1 & TPPFeCI & UV-Lamp/365/6 & TEA & Yes & 48 & 99.0 & 96.0 & 95.0 \\
\hline
\end{tabular}




\begin{tabular}{|c|c|c|c|c|c|c|c|c|c|}
\hline 15 & 1 & TPPFeCl & UV-Lamp/254/6 & TEA & Yes & 24 & 0 & - & 0 \\
\hline
\end{tabular}

* Reactions conditions following general procedure (see 5.1) except specially indicated in Table 1

Study further expanded the scope of the substrate, respectively using 4-methyl cyclohexene, tetramethyl ethylene and styrene. They were on behalf of high sterically hindered olefins, electron-rich branced olefins, electron-deficient conjugated olefins. The experimental results are shown in table 1 (Entry 11-13). They all obtained products with good or excellent selctivity, but electron-deficient conjugated olefin 4 was less active than electron-rich branced olefins 1 to 3 , and the steric effect was not remarkble.
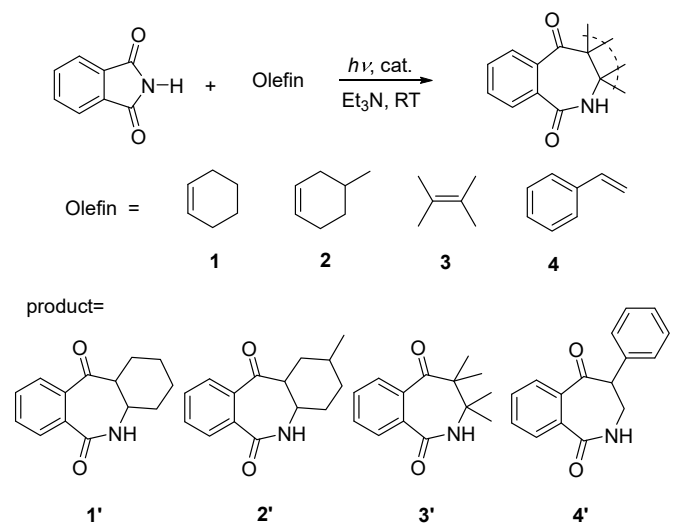

Fig. 2. The synthesis of product 1', 2', 3', 4'.

The research further investigated the specific absorption wavelength for the photoamination to increase light efficiency. The UV-Lamp-365 and UVLamp-254 were used as the light sources respectively. The results showed that $99.0 \%$ conversion and $96.0 \%$ selectivity were achieved with the UV-Lamp-365 (Entry 14), while the UV-Lamp-254 unsuccessfully initiating the reaction (Entry 15), indicated that $365 \mathrm{~nm}$ is specified wavelenth for this reaction.

\section{Conclusions}

Photocatalytic C-N addition amination of phthalimide and olefins using porphyrin iron (III) or phthalocyanine iron(II) as photosensitizer was investigated under ultraviolet light, at temperature of $30{ }^{\circ} \mathrm{C}$, and was conducted successfully. The results showed that the reactions selectively developed $\mathrm{C}-\mathrm{N}$ addition and generated a series of compounds containing benzazepine structure with the highest yield of $99.0 \%$. The scope of substrates was further examined and the results demonstrated that the reaction was effective to various olefins, especially to aliphatic olefins. The optimal reaction conditions were as follows: the molar ratio of PHT to olefin is $1: 10$, the reaction temperature is $30^{\circ} \mathrm{C}$. The simple, low-power, wavelenth-definite insect trap lamp (6 W, $365 \mathrm{~nm}$ ) was used as the light source for the reaction, and a high yield of $95.0 \%$ was obtained. Therefore, this kind of reaction provides a green and efficient method for the synthesis of compounds containing benzazepine structure by the $\mathrm{C}-\mathrm{N}$ addition amination.

\section{Experimental}

\subsection{General procedure for reaction experiment}

Add PHT (200 mg, $1.36 \mathrm{mmol})$, cyclohexene (1.5 mL, $14.6 \mathrm{mmol})$, catalyst $(2 \mathrm{mg})$, TEA $(0.5 \mathrm{~mL})$ and water $(3$ $\mathrm{mL})$ ) in a quartz photo-reactor filled with N2. Keep the reactor at $30{ }^{\circ} \mathrm{C}$ and irradiated by the definite lamp for a period of time. After the reaction, the solvent and excess olefins were removed by distillation under reduced pressure, and the crude product was extracted with dichloromethane and purified by chromatographic column.

\subsection{Synthesis of benzo[b,e]azepine-6,11(2H)- dione 1'}

The reaction was following the general procedure using TPPFeCl as catalyst under UV-Lamp-365 for $48 \mathrm{~h}$. $1 \mathrm{H}$ NMR (400 MHz, CDCl3) $\delta$ (ppm): $7.92(\mathrm{~d}, \mathrm{~J}=7.4,1 \mathrm{H})$, $7.64 \sim 7.55$ (m, $3 \mathrm{H}), 6.43$ (br. s, $1 \mathrm{H}), 4.24$ (s, $1 \mathrm{H}), 2.56$ $(\mathrm{dt}, \mathrm{J}=12.0,3.1 \mathrm{~Hz}, 1 \mathrm{H}), 1.96 \sim 1.35(\mathrm{~m}, 8 \mathrm{H})$.

\subsection{Synthesis of 2-methyl-1,3,4,4a,5,11a- hexahydro-6H-dibenzo[b,e] azepine-6,11(2H)- dione 2'}

The reaction was following the general procedure using TPPFeCl as catalyst under UV-lamp/250-450. 1H-NMR (400 MHz, CDCl3) $\delta(\mathrm{ppm}): 7.90(\mathrm{~d}, \mathrm{~J}=6.3 \mathrm{~Hz}, 1 \mathrm{H})$, 7.62 7.56 (m, $3 \mathrm{H}), 6.55$ (br. s, $1 \mathrm{H}), 4.22$ (s, $1 \mathrm{H}), 2.61$ $(\mathrm{dt}, \mathrm{J}=11.5,3.5 \mathrm{~Hz}, 1 \mathrm{H}), 1.71 \sim 1.16(\mathrm{~m}, 7 \mathrm{H}), 1.02$ (d, J $=6.5,3 \mathrm{H})$.

\subsection{Synthesis of 3,3,4,4-tetramethyl-3,4-dihydro- 1H-benzo[c]azepine-1,5(2H)-dione 3'}

The reaction was following the general procedure using TPPFeCl as catalyst under UV-lamp/250-450. 1H-NMR (400 MHz, CDCl3) $\delta(\mathrm{ppm}): 8.07(\mathrm{~m}, 1 \mathrm{H}, 6-\mathrm{H}), 7.87$ (m, $1 \mathrm{H}, 9-\mathrm{H}), 7.58$ (m, $2 \mathrm{H}), 6.89$ (br. d, $1 \mathrm{H}), 1.26$ (s, 6 $\mathrm{H}), 1.24(\mathrm{~s}, 6 \mathrm{H})$.

\subsection{Synthesis of 4-phenyl-3,4-dihydro-1H- benzo[c]azepine-1,5(2H)-dione 4'}

The reaction was following the general procedure using TPPFeCl as catalyst under UV-lamp/250-450. 1H-NMR $(400 \mathrm{MHz}, \mathrm{CDCl} 3) \delta(\mathrm{ppm}): 7.96(\mathrm{dd}, \mathrm{J}=1.1,7.6 \mathrm{~Hz}, 1$ H, 6-H), 7.73 7.56 (m, $3 \mathrm{H}), 7.35 \sim 7.13(\mathrm{~m}, 5 \mathrm{H}), 4.17$ $(\mathrm{dd}, \mathrm{J}=3.9,10.2 \mathrm{~Hz}, 1 \mathrm{H}), 3.85$ (ddd, $\mathrm{J}=5.9,10.2,15.0$ $\mathrm{Hz}, 1 \mathrm{H}), 3.67$ (ddd, J = 4.0, 6.8, $15.1 \mathrm{~Hz}, 1 \mathrm{H})$. 


\section{Acknowledgments}

The authors are grateful to Zhejiang Jianye Chemical Co., Ltd. for financial support.

\section{References}

1. Z. Zhang, S. Yong, J. Wu, et al. Org. Lett, 18, 3038 (2016)

2. B. Edward, W. Duncan, Org. Lett, 12, 5330 (2010)

3. S. Dipesh , P. Benudhar. Adv. Synth. Catal, 361, 1(2019)

4. O. Lourdes, C. Luis, D. Domingo, Tetrahedron, 55, 4445(1999)

5. S.F. Martin, Pergamon Press, 30, 251(1988)

6. S. Lesley, G. Karen, Drugs, 60, 1095 (2000).

7. S. Ralph, Y. Cheol, J. Kyung, Tetrahedron, 57, 7785(2001)

8. M. Hari, K. Sreenivasulu, Synthetic Communications, 31, 3005(2001)

9. M. Thomas, H. Kai, Chemical Reviews, 108, 3795(2008).

10. N. Thomas, W. Vijay, G. Abhijit, M. Rashimi, Org. Lett, 7, 4967(2005)

11. H. Laszlo, M. Tibor. ApplCatalA, 296, 209(2005)

12. C. Sanchez, E. Perez, R. Garcia, Tetrahedron, 58, 7267(2002)

13. A. Alan, L. Frederick, S. Willian, Journal of the American Chemical Society, 86, 3145(1964)

14. Nevin W A, Liu W, M. Melnik, et al, Journal of Electroanalytical Chemistry, 213, (1986) 